\title{
Demanda psicossocial e demanda física no trabalho: iniquidades segundo raça/cor
}

\author{
Physical and psychosocial demand at work: inequities related to \\ race/skin color
}

Janaína Santos de Siqueira (https://orcid.org/0000-0001-6439-5265) ${ }^{1}$

Rita de Cássia Pereira Fernandes (https://orcid.org/0000-0002-3353-5365) ${ }^{2}$

${ }^{1}$ Programa de PósGraduação em Saúde, Ambiente e Trabalho, Universidade Federal da Bahia (UFBA). Largo do Terreiro de Jesus $\mathrm{s} / \mathrm{n}$, Centro Histórico. 40026010 Salvador BA Brasil. js.desiqueira@gmail.com ${ }^{2}$ Faculdade de Medicina da Bahia, UFBA. Salvador BA Brasil.

\begin{abstract}
This cross-sectional study investigated the association between self-reported race/skin color and two outcomes - psychosocial demand and physical demand at work - in 1,032 workers in an urban cleaning services company and two footwear manufacturers, located in the State of Bahia, Brazil. Psychosocial demand was measured through the Job Content Questionnaire and physical demand was measured through questions about postures and cargo handling. A Cox regression analysis provided prevalence ratios (PR) adjusted by age, gender, and educational level. Among blacks, there is a higher proportion of garbage collectors and a lower proportion of supervisory positions. Black workers are more subject to high psychological demand and low job control and, consequently, to high strain $(P R=1.65)$. Also, they are more exposed to work with arms above shoulder level $(P R=1.93)$, and material handling $(P R=1.62)$, compared to white workers. Brown workers are more exposed to low job control $(P R=1.36)$, work with arms above shoulder level $(P R=1.48)$, and material handling $(P R=1.25)$, also compared with whites. Social support is lower among blacks and brown. The study demonstrated inequities in psychosocial and physical exposures at work that are in line with the structural conception of racism. This evidence can contribute to the adoption of practices that increase equity in the world of work.
\end{abstract}

Key words Psychosocial factors, Physical effort, Inequities, Racism, Worker's health
Resumo Este estudo de corte transversal investigou a associação entre raça/cor autorrelatada e dois desfechos-demanda psicossocial e demanda física no trabalho - em 1.032 trabalhadores da Limpeza Urbana e da Indústria Calçadista, na Bahia. Mensurou-se demanda psicossocial por meio do Job Content Questionnaire e demanda física, com questões sobre posturas e manuseio de carga. Regressão de Cox forneceu razões de prevalência (RP) ajustadas por idade, sexo e escolaridade. Entre os pretos há maior proporção de coletores de lixo e menor proporção de cargos de supervisão. Trabalhadores pretos estão mais submetidos à alta demanda psicológica e à baixo controle e, consequentemente, à mais alta exigência no trabalho $(R P=1,65)$. Ademais, são mais expostos ao trabalho com braços elevados $(R P=1,93)$ e ao manuseio de carga $(R P=1,62)$, comparados com brancos. Pardos estão mais expostos ao baixo controle $(R P=1,36)$, ao trabalho com braços elevados $(R P=1,48)$ e com manuseio de carga $(R P=1,25)$, também comparados com brancos. Apoio social é mais baixo entre os pretos e pardos. O estudo demonstrou iniquidades nas exposições psicossocial e física no trabalho que estão em acordo com a concepção estrutural do racismo e sua evidência pode contribuir para condutas que ampliem a equidade no mundo do trabalho.

Palavras-chave Fatores psicossociais, Esforço físico, Iniquidades, Racismo, Saúde do trabalhador 


\section{Introdução}

O mundo do trabalho é marcado por desigualdades e iniquidades. Enquanto as primeiras decorrem da variabilidade de características humanas, as iniquidades consistem em diferenças injustamente produzidas, evitáveis e passíveis de extinção que vulnerabilizam grupos na sociedade. São geralmente mantidas por relações de poder ${ }^{1-3}$.

O racismo estrutural, presente na sociedade e, por conseguinte, nas instituições, contribui para a manutenção de desvantagens entre grupos étnicos historicamente subjugados, sustentando iniquidades ${ }^{4-6}$.

A concentração de pior posição socioeconômica entre pretos ou pardos, frequentemente compreendidos na literatura como negros devido ao histórico comum de vulnerabilidade ${ }^{7-9}$, é um dos efeitos do racismo estrutural, podendo repercutir nas exposições ocupacionais ${ }^{4,6,9,10}$. Ser negro foi associado ao trabalho em ocupações de alto risco para ocorrência de lesões não-fatais e adoecimento nos Estados Unidos ${ }^{11}$. Ademais, ocupações nas quais há maior proporção de negros estão associadas aos piores autorrelatos do estado de saúde por trabalhadores negros e não negros ${ }^{12}$.

No contexto de inserção ocupacional no Brasil, tarefas com menor autonomia e prestígio e maior esforço físico são frequentes entre trabalhadores negros. $\mathrm{O}$ trabalho doméstico foi destacado entre mulheres negras ocupadas $(21,8 \%$ entre negras versus $12,6 \%$ entre brancas) $)^{8}$, assim como o trabalho informal, mais precário, foi superior entre negros $(47,3 \% \text { versus } 34,6 \%)^{9}$, enquanto brancos predominam entre magistrados $(82,8 \%)^{13}$ e em cargos gerenciais $(68,6 \%)^{9}$. Este panorama evidencia uma hierarquização racial no trabalho ${ }^{6,14}$.

Nesta perspectiva, a raça é compreendida como um produto das relações históricas, socioculturais e de ancestralidade, que contribuem para a formação identitária, contrapondo-se às identidades baseadas em diferenciações genéticas $^{15-19}$ e é utilizada em estudos epidemiológicos para operacionalização do construto "etnia".

Este estudo parte da hipótese de que existem no mundo do trabalho, embora sejam escassamente objeto de investigação, iniquidades raciais relativas à exposição ocupacional, isto é, a maior sobrecarga física e psicossocial deve ser encontrada em tarefas desempenhadas por pretos e pardos, expressando as condições adversas no mundo do trabalho.

O estudo da demanda psicossocial no trabalho vem sendo feito com base em modelos teóricos, dentre os quais o Modelo DemandaControle (MDC) ${ }^{20}$ preconiza que os aspectos psicossociais podem atuar como estressores, constituindo fatores de risco para o adoecimento físico e psíquico. Este modelo define duas dimensões: psicológica, que consiste na exigência psicológica requerida no curso de uma tarefa, e o controle no trabalho, que envolve o uso de habilidades e criatividade pelo trabalhador, além da autonomia da qual dispõe para condução do trabalho. O modelo descreve quatro experiências no trabalho conforme níveis de exposição à demanda psicológica e ao controle. O trabalho de alta exigência, que resulta de alta demanda psicológica combinada a um baixo controle, relaciona-se com desmotivação, baixa autoestima e perda de habilidades no trabalho ${ }^{20}$, e é a experiência mais associada a agravos à saúde quando comparada com as demais situações no trabalho: trabalho de baixa exigência, trabalho passivo e trabalho ativo ${ }^{20}$.

Este MDC foi ampliado, passando a incorporar a dimensão apoio social, proveniente de colegas e de supervisor, sendo esta entendida como a dimensão que pode modificar a experiência de alta exigência, protegendo o trabalhador que conta com alto apoio social ${ }^{21}$.

Existem evidências de que pretos e pardos estejam mais expostos, principalmente, ao baixo controle e à alta demanda, contudo a literatura científica sobre o tema é escassa ${ }^{10,22-24}$.

Além de maior demanda psicossocial, é possível que trabalhadores pretos e pardos estejam mais submetidos à demanda física no trabalho, que envolve posturas penosas de corpo inteiro ou de segmentos corporais, além de manuseio de carga e uso de força ${ }^{25}$, por exemplo, associados, entre outros agravos, aos distúrbios musculoesqueléticos e, particularmente, à lombalgia ocupacional $^{26-30}$. Esta situação foi verificada entre trabalhadores afro-americanos, que realizam mais tarefas envolvendo agachamentos e manuseio de carga, contribuindo para a maior percepção de dor quando comparados com os brancos ${ }^{31}$.

Assim, o presente estudo objetivou analisar a associação entre raça/cor e dois desfechos do trabalho que são as demandas psicossociais e as físicas a que estão submetidos os trabalhadores.

\section{Método}

Realizou-se estudo de corte transversal com trabalhadores provenientes da Limpeza Urbana e da Indústria de Calçados no estado da Bahia. Este pool de inquéritos foi concebido em projeto de 
cooperação internacional ${ }^{32,33}$, no qual a estratégia de reunião dos inquéritos, conduzidos com os mesmos procedimentos e técnicas, visou assegurar o maior número de participantes e favorecer a variabilidade de exposição ocupacional, de interesse analítico.

O inquérito com trabalhadores da Limpeza Urbana, todos do sexo masculino, na capital, foi um censo $(n=624)$ realizado em 2010 . O segundo inquérito, com trabalhadores de duas empresas calçadistas do interior do Estado, utilizou amostra aleatória estratificada proporcional por sexo e empresa $(n=446)$, em 2012. Os trabalhadores da Limpeza Urbana estavam alocados em ocupações de operação e manutenção, enquanto os calçadistas estavam em ocupações administrativas, operacionais e de manutenção. As ocupações mais frequentes foram as de coletores, agentes de limpeza e as relacionadas com atividades operacionais da fabricação de calçados. No conjunto dos dois inquéritos, a taxa de respostas foi de 97\%. Ambos os inquéritos foram realizados com base nos pressupostos da Vigilância em Saúde do Trabalhador, que indicavam sua oportunidade, vinculando-se a intervenções nas condições de trabalho, em cooperação com a rede de serviço em Saúde do Trabalhador, na Bahia.

\section{Procedimentos e instrumento de coleta de dados}

O questionário foi aplicado nos locais de trabalho, durante a jornada, assegurando privacidade, por equipe de entrevistadores treinados, composta por cinco mestrandos (três fisioterapeutas, um engenheiro de segurança, um ergonomista) e quatro acadêmicos de graduação de fisioterapia. Todos os trabalhadores foram esclarecidos sobre os objetivos do estudo, com garantia da confidencialidade das informações.

Das questões do questionário, foram utilizadas neste estudo, as sociodemográficas - idade, sexo e escolaridade - e as ocupacionais, relativas ao Job Content Questionnaire (JCQ) ${ }^{34,35}$ e às questões de demanda física.

No intuito de minimizar o efeito sobrevivência do trabalhador sadio (que pode resultar da exclusão daqueles incapacitados temporariamente para o trabalho), todos os trabalhadores empregados no momento do estudo, com no mínimo 12 meses de trabalho, foram elegíveis. Indivíduos afastados por evento supostamente não relacionado ao trabalho, a exemplo de licença gestacional, uma vez sorteados, foram substituídos pelo próximo nome da lista nominal de trabalhadores fornecida pela respectiva empresa. Houve uma entrevista realizada em domicílio em trabalhador da Indústria de Calçados que se encontrava em licença-médica. Não houve afastamento de longa duração do trabalho, entre os respondentes.

\section{Variáveis dependentes}

\section{Demanda psicossocial no trabalho}

Os aspectos psicossociais do trabalho de acordo com o Modelo Demanda-Controle e Apoio Social (MDC-AS) ${ }^{20,21}$ que caracterizaram a demanda psicossocial foram demanda psicológica, controle sobre o trabalho, apoio social e experiência de alta exigência no trabalho, mensurados através do $\mathrm{JCQ}^{34,35}$. Os quadrantes do MDC descrevem formas distintas de exposição psicossocial. Altos controle e demanda constituem o trabalho ativo, baixos controle e demanda configuram o trabalho passivo, baixa demanda e alto controle resultam em trabalho com baixa exigência, e, por fim, alta demanda e baixo controle caracterizam o trabalho de alta exigência, considerada a experiência mais deletéria ${ }^{20}$.

Apoio social proveniente de supervisor e de colegas, somados, compuseram a dimensão do apoio social.

A escala de respostas ao JCQ é ordinal do tipo Likert com quatro pontos, variando de "discordo fortemente" (1) a "concordo fortemente" (4).

A validade e reprodutibilidade do JCQ foram avaliadas como boa em estudo conduzido com trabalhadores formais e informais ${ }^{35}$ e Santos et al. ${ }^{36}$ confirmaram a dimensionalidade do JCQ, conforme o MDC-AS.

\section{Demanda física no trabalho}

Foi investigada por meio de seis questões sobre posturas gerais ou de segmentos corporais (em pé, andando, agachado, com tronco em rotação, com braços acima da altura dos ombros, pressão física com as mãos sobre objeto de trabalho) e três sobre manuseio de carga (levantar, empurrar e puxar). Escala de resposta numérica, de seis pontos ( 0 a 5 ), foi utilizada com qualificadores de duração nas extremidades (0-“jamais" e 5-"o tempo todo"), exceto para o item sobre uso de pressão física com as mãos, cuja escala de resposta foi de intensidade (0-"muito fraca" e 5-"muito forte"). Validade e confiabilidade destes itens de demanda física foram testadas por Fernandes et al. ${ }^{37}$ na população do presente estudo. Os resultados evidenciam dimensionalidade $\mathrm{e}$ confiabilidade variando de excelente a moderada 
para a maior parte dos itens, o que recomenda o uso das questões em estudos epidemiológicos.

O uso das questões sobre demanda física visa superar a mensuração de exposição baseada no título de ocupação, permitindo incorporar a variabilidade de exposição entre sujeitos sob um mesmo título, dependendo das tarefas efetivamente realizadas. Portanto, o autorrelato de trabalhadores tem sido considerada medida com maior validade na avaliação da exposição física ${ }^{37}$.

\section{Variável independente principal}

A variável "raça/cor" foi obtida através do autorrelato, conforme categorias propostas pelo Instituto Brasileiro de Geografia e Estatística (IBGE): branco, preto, amarelo, pardo e indíge$\mathrm{na}^{7}$. Foram covariáveis de interesse: idade, sexo e escolaridade

\section{Abordagem estatística dos dados}

\section{Abordagem descritiva}

Foram obtidos os valores mínimo e máximo, as médias e medianas das variáveis quantitativas e as frequências absolutas e relativas das variáveis qualitativas.

Cinco grupos ocupacionais a partir da noção de grupos homogêneos de exposição, foram definidos com base nas semelhanças das tarefas desenvolvidas e na posição na estrutura organizacional. São estes: coletor/agente de limpeza; motorista da Limpeza Urbana; coordenador/ supervisor/cargo administrativo; trabalhadores operacionais e de fabricação de calçados; trabalhadores de manutenção. A distribuição dos trabalhadores segundo raça/cor em cada um dos grupos ocupacionais foi apresentada. A descrição das tarefas realizadas em cada grupo ocupacional não é objeto deste artigo $^{32}$.

Os escores obtidos das dimensões do MDC -AS foram descritos por meio da mediana e dos valores máximo e mínimo. Em seguida, "demanda psicológica" e "controle sobre o trabalho" foram dicotomizadas em alta ou baixa exposição, a partir da mediana, assim como o escore obtido na dimensão "apoio social", que soma o apoio de supervisor e de colegas.

As variáveis categóricas do MDC-AS, deman$\mathrm{da}$, controle e apoio social, foram descritas em separado. Em seguida, os quadrantes do MDC foram utilizados e o trabalho de alta exigência foi o estrato de risco a ser comparado com ausência de alta exigência, que agregou os demais quadrantes, trabalho passivo, ativo e de baixa exi- gência, sendo descritos de acordo com a variável raça/cor.

As respostas ao questionário sobre demanda física no trabalho foram categorizadas, sendo que aquelas de zero a dois na escala corresponderam a "baixa exposição" e de três a cinco a "alta exposição”. A exposição à demanda física foi descrita para cada item, de acordo com a raça/cor.

Raça/cor foi categorizada em "branco", "preto" e "pardo". Trabalhadores que se autoclassificaram amarelos $(n=15)$ ou indígenas $(n=20)$ foram excluídos da população do estudo, uma vez que apresentaram número bastante reduzido, que inviabilizou a análise em estratos separados da variável independente principal nas modelagens. A incorporação dos indígenas ao estrato de pretos e dos amarelos ao estrato de brancos, a fim de não os excluir da análise, foi explorada pelas autoras, tendo mostrado resultados semelhantes nas modelagens, conforme previsto, tendo em vista o baixo número de indivíduos em cada grupo, no entanto, a decisão pela exclusão baseou-se na crítica a uma heteroclassificação.

\section{Abordagem analítica}

A categoria "branco" da variável independente raça/cor foi considerada o estrato de referência (não-exposto) nas análises.

A variável "idade" foi dicotomizada a partir da mediana (31 anos) e a "escolaridade" foi estratificada em menor e maior ou igual a "ensino médio completo".

As associações analisadas foram apresentadas por meio de razões de prevalência (RP) fornecidas pela Regressão de Cox para estudos transversais $^{38,39}$.

Para a modelagem, além da variável independente principal (raça/cor), foram inseridas as variáveis sexo, idade e escolaridade, selecionadas com base nas evidências da literatura. $\mathrm{O}$ primeiro modelo, para cada um dos dois desfechos, apresenta a medida bruta da associação principal (RP), resultado da análise univariada. No segundo modelo, a RP é ajustada por sexo e idade (mulheres e jovens foram os estratos de exposição $)^{40}$. O terceiro modelo, além das variáveis já inseridas, incluiu-se a variável "escolaridade", obtendo-se RP ajustada pelas três covariáveis, que compuseram os modelos finais.

Considerando que a população deste estudo não é uma amostra aleatória, evitou-se o uso impróprio, embora habitual, da abordagem inferencial, quando esta é realizada em estudos sem amostra aleatória ou em censos. Trata-se de estudo analítico, no qual adotaram-se procedimen- 
tos compatíveis com a natureza não aleatória da população investigada, conforme recomenda a literatura especializada sobre o tema ${ }^{41-43}$. Assim, da análise multivariada são apresentadas as variáveis com suas respectivas medidas de associação (razão de prevalência) que caracterizam o estudo analítico.

A análise dos dados foi realizada através do software Statistical Package for Social Sciences (SPSS), versão 21.0.

\section{Aspectos éticos}

O projeto de pesquisa do qual deriva o estudo foi aprovado pelo Comitê de Ética em Pesquisa da Faculdade de Medicina da Universidade Federal da Bahia, estando de acordo com a Resolução no 466/2012 do Conselho Nacional de Saúde. Todos os participantes dos inquéritos assinaram o Termo de Consentimento Livre e Esclarecido.

\section{Resultados}

Foram estudados 1.032 trabalhadores, entre os quais predominam pretos $(47,4 \%$; $\mathrm{n}=489)$ seguidos por pardos $(42,7 \% ; n=441)$ e brancos $(9,9 \%$; $\mathrm{n}=102$ ). Houve dado perdido para raça/cor de três indivíduos.

A mediana da idade correspondeu a 31 anos e o sexo masculino representou 78,7\% (842). Parcela expressiva dos trabalhadores não concluiu o ensino médio $(46,0 \% ; n=490)$. Brancos apresentaram maior escolaridade $(62,7 \%)$, ou seja, ensino médio completo ou universitário, seguidos por pardos $(58,0 \%)$ e pretos $(48,6 \%)$.

A Tabela 1 apresenta a distribuição dos grupos ocupacionais, segundo raça/cor. Entre os pretos há maior proporção de "coletor de lixo urbano"
$(57,5 \%)$ e menor proporção em cargos de supervisão, coordenação e trabalho administrativo $(3,5 \%)$, ao passo que entre os brancos, $23,5 \%$ são coletores e 20,6\% ocupam funções de supervisão e administrativas. Enquanto 17,6\% dos brancos são motoristas da Limpeza Urbana, este percentual é de aproximadamente 8,0\% entre pretos.

A Tabela 2 apresenta as variáveis referentes à exposição psicossocial no trabalho de acordo com o MDC-AS, estratificadas por raça/cor. Indivíduos pretos apresentaram maior demanda psicológica e menor controle sobre o trabalho e os brancos, as medidas mais favoráveis, maior controle e menor demanda psicológica. A prevalência de alta exigência no trabalho foi superior entre pretos. Observou-se entre esses a maior frequência de baixo apoio social de supervisores, entretanto, a maior frequência de alto apoio de colegas.

A análise multivariada mostrou que, comparados aos brancos, pretos apresentaram 65\% a mais na prevalência de alta exigência e $60 \%$ a mais na prevalência de baixo controle no trabalho, em modelos ajustados por idade, sexo e escolaridade. Alta demanda psicológica e baixo apoio social também foram mais prevalentes entre os pretos, porém essas associações tiveram menor magnitude. Entre pardos, a prevalência de baixo controle foi $36 \%$ a mais em comparação com brancos (Tabela 3 ).

A Tabela 4 apresenta a distribuição dos itens de demanda física no trabalho, segundo raça/cor. Exposição ao trabalho em pé foi mais frequente entre pretos. Nos demais itens, observam-se maiores frequências de exposição entre pretos, seguidos por pardos, e com menores frequências estão os brancos.

Foi verificada associação positiva entre demanda física, para todos os itens, e raça/cor preta e as associações se mantiveram após ajuste por

Tabela 1. Distribuição dos trabalhadores da Limpeza Urbana e da Indústria Calçadista por grupo ocupacional em cada categoria da variável raça/cor na Bahia.

\begin{tabular}{|c|c|c|c|c|c|c|}
\hline \multirow{4}{*}{ Raça/cor } & \multicolumn{5}{|c|}{ Grupos Ocupacionais } & \multirow[b]{2}{*}{ Total } \\
\hline & $\begin{array}{c}\text { Coletor/Agente } \\
\text { de Limpeza } \\
\text { Urbana }\end{array}$ & $\begin{array}{l}\text { Motorista de } \\
\text { caminhão }\end{array}$ & Manutenção & $\begin{array}{c}\text { Supervisor/ } \\
\text { Coordenador/ } \\
\text { Administrativo }\end{array}$ & $\begin{array}{l}\text { Fabricação de } \\
\text { calçados* }\end{array}$ & \\
\hline & $n=437$ & $n=112$ & $\mathrm{n}=\mathbf{8 3}$ & $\mathrm{n}=\mathbf{8 4}$ & $n=316$ & $\mathrm{n}=1.032$ \\
\hline & $42,4 \%$ & $10,9 \%$ & $8,0 \%$ & $8,1 \%$ & $30,6 \%$ & $100 \%$ \\
\hline Preto & $281(57,5 \%)$ & $40(8,2 \%)$ & $35(7,1 \%)$ & $17(3,5 \%)$ & $116(23,7 \%)$ & 489 \\
\hline Pardo & $132(29,9 \%)$ & $54(12,3 \%)$ & $42(9,5 \%)$ & $46(10,4 \%)$ & $167(37,9 \%)$ & 441 \\
\hline Branco & $24(23,5 \%)$ & $18(17,6 \%)$ & $6(5,9 \%)$ & $21(20,6 \%)$ & $33(32,4 \%)$ & 102 \\
\hline
\end{tabular}

^Operador na produção de calçados: Montador de calçados/Operador de máquinas/Costureiro. 
Tabela 2. Demanda psicossocial no trabalho referente ao Modelo Demanda, Controle e Apoio Social (MDC-AS) segundo raça/cor de trabalhadores da Limpeza Urbana e da Indústria Calçadista na Bahia.

\begin{tabular}{|c|c|c|c|}
\hline \multirow{2}{*}{$\begin{array}{c}\text { Demanda psicossocial no trabalho } \\
\text { (MDC-AS) }\end{array}$} & \multicolumn{3}{|c|}{ Raça/Cor } \\
\hline & Preto & Pardo & Branco \\
\hline \multicolumn{4}{|l|}{ Demanda psicológica } \\
\hline Mediana (mín.-máx.)* & $36,0(20-48)$ & $34,0(16-48)$ & $34,5(16-48)$ \\
\hline Baixa n (\%) & $208(42,5)$ & $259(58,7)$ & $54(53,0)$ \\
\hline Alta n (\%) & $281(57,5)$ & $182(41,3)$ & $48(47,0)$ \\
\hline \multicolumn{4}{|l|}{ Controle } \\
\hline Mediana (mín.-máx.)* & $58,0(26-88)$ & $60,0(32-86)$ & $64,0(40-94)$ \\
\hline Baixo n $(\%)$ & $287(58,7)$ & $231(52,4)$ & $39(38,2)$ \\
\hline Alto $\mathrm{n}(\%)$ & $202(41,3)$ & $210(47,6)$ & $63(61,8)$ \\
\hline \multicolumn{4}{|l|}{ Apoio social do supervisor } \\
\hline Mediana (mín.-máx.)* & $11(03-16)$ & $11(05-16)$ & $11(04-16)$ \\
\hline Baixo n (\%) & $323(66,1)$ & $266(60,3)$ & $58(56,9)$ \\
\hline Alto n (\%) & $166(33,9)$ & $175(39,7)$ & $44(43,1)$ \\
\hline \multicolumn{4}{|l|}{ Apoio social dos colegas } \\
\hline Mediana (mín.-máx.)* & $12(04-16)$ & $12(06-16)$ & $12(04-16)$ \\
\hline Baixo n (\%) & $272(55,6)$ & $296(67,1)$ & $64(62,8)$ \\
\hline Alto n (\%) & $217(44,4)$ & $145(32,9)$ & $38(37,2)$ \\
\hline \multicolumn{4}{|l|}{ Apoio social (supervisor e colegas) } \\
\hline Mediana (mín.-máx.)* & $23,0(10-32)$ & $23,0(12-32)$ & $23,0(12-32)$ \\
\hline Baixo n (\%) & $291(59,9)$ & $257(58,3)$ & $51(50,0)$ \\
\hline Alto n (\%) & $195(40,1)$ & $184(41,7)$ & $51(50,0)$ \\
\hline \multicolumn{4}{|l|}{ Alta exigência n (\%) } \\
\hline Sim & $186(38,0)$ & $114(25,9)$ & $23(22,5)$ \\
\hline Não & $303(62,0)$ & $327(74,1)$ & $79(77,5)$ \\
\hline
\end{tabular}

*Valores mínimo e máximo obtidos para a mediana em cada dimensão do Modelo Demanda, Controle e Apoio Social.

Fonte: Elaborado pelas autoras.

Tabela 3. Associação entre demanda psicossocial no trabalho, de acordo com o Modelo Demanda, Controle e Apoio Social, e raça/cor em trabalhadores da Limpeza Urbana e da Indústria Calçadista, na Bahia.

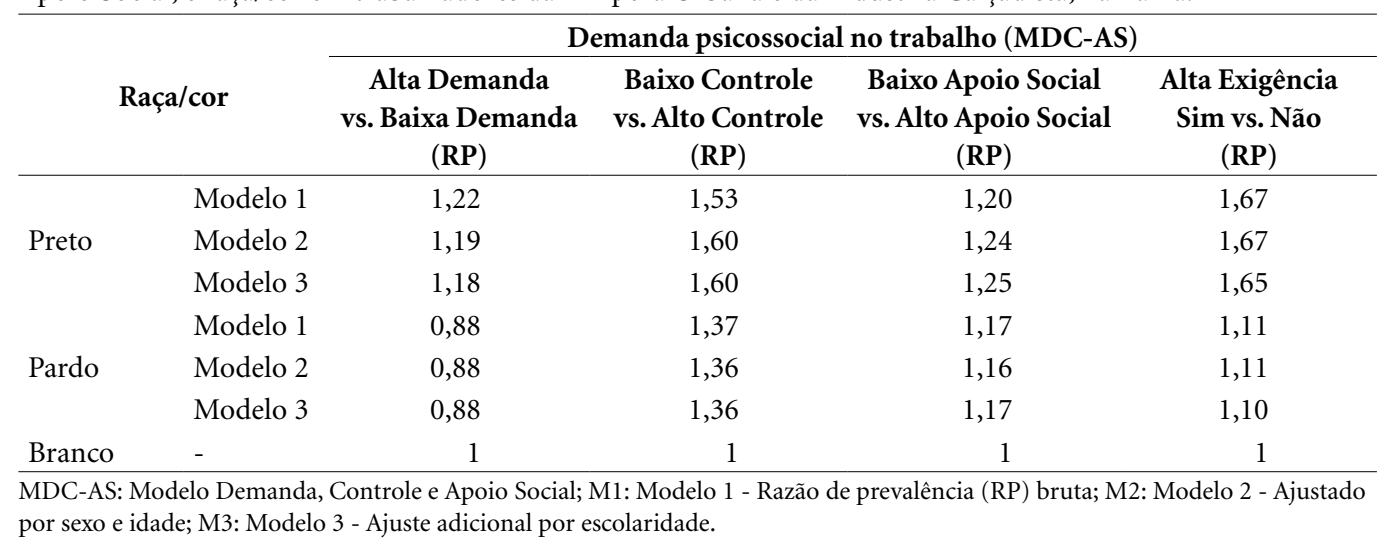

Fonte: Elaborado pelas autoras.

sexo, idade e escolaridade. Destacaram-se o trabalho com braço elevado e em postura agachada, além do manuseio de carga, com prevalências de alta exposição, no mínimo, $44 \%$ superiores entre pretos comparados aos brancos (Tabela 5).
Pardos, comparados aos brancos, apresentaram maiores exposições em todos os itens de demanda física, mesmo após ajuste, porém as associações tiveram menor magnitude do que quando se comparam pretos aos brancos. Em- 
Tabela 4. Distribuição da exposição à demanda física no trabalho segundo raça/cor, em trabalhadores da Limpeza Urbana e da Indústria Calçadista, na Bahia.

\begin{tabular}{|c|c|c|c|c|c|c|}
\hline \multirow{4}{*}{ Demanda Física no Trabalho } & \multicolumn{6}{|c|}{ Raça/cor } \\
\hline & \multicolumn{2}{|c|}{ Preto } & \multicolumn{2}{|c|}{ Pardo } & \multicolumn{2}{|c|}{ Branco } \\
\hline & $\begin{array}{c}\text { Baixa } \\
\text { Exposição }\end{array}$ & $\begin{array}{c}\text { Alta } \\
\text { Exposição }\end{array}$ & $\begin{array}{c}\text { Baixa } \\
\text { Exposição }\end{array}$ & $\begin{array}{c}\text { Alta } \\
\text { Exposição }\end{array}$ & $\begin{array}{c}\text { Baixa } \\
\text { Exposiçãoo }\end{array}$ & $\begin{array}{c}\text { Alta } \\
\text { Exposição }\end{array}$ \\
\hline & n (\%) & n (\%) & n (\%) & n (\%) & n (\%) & n (\%) \\
\hline \multicolumn{7}{|l|}{$\begin{array}{l}\text { Posturas no trabalho (geral e de } \\
\text { segmentos corporais) }\end{array}$} \\
\hline Em pé & $85(17,5)$ & $400(82,5)$ & $143(32,6)$ & $295(67,4)$ & $35(34,3)$ & $66(64,7)$ \\
\hline Andando & $226(46,9)$ & $256(53,1)$ & $244(55,7)$ & $194(44,3)$ & $59(57,8)$ & $42(42,2)$ \\
\hline Agachado & $215(44,5)$ & $268(55,5)$ & $283(65,1)$ & $152(34,9)$ & $68(66,6)$ & $33(33,4)$ \\
\hline Com tronco em rotação & $120(24,8)$ & $363(75,2)$ & $169(38,7)$ & $268(61,3)$ & $48(47,0)$ & $54(53,0)$ \\
\hline Com braços acima dos ombros & $241(50,0)$ & $241(50,0)$ & $298(67,9)$ & $141(32,1)$ & $81(79,4)$ & $21(20,6)$ \\
\hline Pressão física com as mãos & $134(27,7)$ & $350(72,3)$ & $164(37,3)$ & $276(62,7)$ & $50(50,0)$ & $50(50,0)$ \\
\hline \multicolumn{7}{|l|}{ Manuseio de carga } \\
\hline Levantar & $161(32,9)$ & $328(67,1)$ & $239(54,2)$ & $202(45,8)$ & $62(60,8)$ & $40(39,2)$ \\
\hline Empurrar & $200(41,0)$ & $287(59,0)$ & $260(59,0)$ & $181(41,0)$ & $69(67,6)$ & $33(32,4)$ \\
\hline Puxar & $211(43,2)$ & $277(56,8)$ & $265(60,1)$ & $176(39,9)$ & $68(66,7)$ & $34(33,3)$ \\
\hline
\end{tabular}

Tabela 5. Associação entre exposição à demanda física no trabalho e raça/cor em trabalhadores da Limpeza Urbana e da Indústria Calçadista, na Bahia.

\begin{tabular}{|c|c|c|c|c|c|c|c|c|c|c|}
\hline \multirow{3}{*}{\multicolumn{2}{|c|}{ Raça/cor }} & \multicolumn{9}{|c|}{ Alta exposição à demanda física no trabalho vs. Baixa exposição (RP) } \\
\hline & & \multicolumn{6}{|c|}{ Posturas gerais ou de segmentos corporais } & \multicolumn{3}{|c|}{ Manuseio de carga } \\
\hline & & Em pé & Andando & Agachado & $\begin{array}{c}\text { Tronco } \\
\text { em } \\
\text { rotação }\end{array}$ & $\begin{array}{c}\text { Braços } \\
\text { acima dos } \\
\text { ombros }\end{array}$ & $\begin{array}{c}\text { Pressão } \\
\text { física com } \\
\text { as mãos }\end{array}$ & Levantar & Empurrar & Puxar \\
\hline \multirow{3}{*}{ Preto } & M1 & 1,26 & 1,25 & 1,70 & 1,42 & 2,43 & 1,42 & 1,71 & 1,82 & 1,70 \\
\hline & M2 & 1,21 & 1,17 & 1,57 & 1,39 & 2,16 & 1,37 & 1,63 & 1,71 & 1,60 \\
\hline & M3 & 1,18 & 1,12 & 1,44 & 1,37 & 1,93 & 1,35 & 1,55 & 1,62 & 1,51 \\
\hline \multirow{3}{*}{ Pardo } & M1 & 1,03 & 1,04 & 1,07 & 1,16 & 1,56 & 1,23 & 1,17 & 1,27 & 1,20 \\
\hline & M2 & 1,05 & 1,06 & 1,10 & 1,16 & 1,61 & 1,23 & 1,18 & 1,30 & 1,22 \\
\hline & M3 & 1,04 & 1,03 & 1,03 & 1,15 & 1,48 & 1,22 & 1,14 & 1,25 & 1,17 \\
\hline Branco & - & 1 & 1 & 1 & 1 & 1 & 1 & 1 & 1 & 1 \\
\hline
\end{tabular}

M1: Modelo 1 - Razão de prevalência (RP) bruta; M2: Modelo 2 - Ajustado por sexo e idade; M3: Modelo 3 - Ajuste adicional por escolaridade.

Fonte: Elaborado pelas autoras.

purrar carga e trabalhar com braços elevados foram $25 \%$ e $48 \%$ mais frequentes, respectivamente, entre pardos (Tabela 5).

\section{Discussão}

Os achados vão ao encontro da hipótese de estudo acerca das iniquidades raciais na exposição ocupacional. Trabalhadores pretos estão subme- tidos a mais altas demandas no trabalho, psicossocial e física. Esses apresentaram, principalmente, menor grau de controle sobre o seu trabalho, além de estarem mais submetidos a alta demanda psicológica e, consequentemente, ao trabalho de alta exigência, comparados com brancos. Estão também mais expostos às posturas corporais anômalas, tais como trabalho com os braços elevados acima dos ombros e em postura agachada, além de realizarem mais manuseio de carga. En- 
tre os pardos, ocorreram, em geral, associações de menor magnitude com as demandas no trabalho, também comparados com brancos.

Portanto, o trabalho físico de maior exigência corporal, também designado como trabalho físico pesado, foi destinado principalmente aos pretos, que ademais, estão submetidos a situações estressoras, com maior exigência de tempo e menor autonomia no desempenho das tarefas.

Trabalhadores pretos predominaram nas ocupações operacionais, mas foram bem menos representados nos cargos de coordenação, supervisão e administração, comparados aos brancos, $3,5 \%$ e $20,6 \%$, respectivamente. Ademais, nas equipes de coleta de lixo, os pretos eram predominantemente coletores em vez de motoristas. Embora a população deste estudo seja relativamente homogênea no que diz respeito ao baixo prestígio social, há diferenças nos níveis de controle sobre o trabalho conforme grupo ocupacional, a exemplo dos motoristas da Limpeza Urbana, com maior proporção entre os brancos e que apresentam maior grau de controle que coletores/agentes de limpeza, estes com maior proporção entre os $\operatorname{pretos}^{28}$. Quanto a este diferencial, estudo sobre o trabalho de equipes de coleta de lixo urbano, baseado na Análise Ergonômica do Trabalho, revelou que motoristas lideravam a equipe de coleta, sendo responsáveis por determinar o ritmo de trabalho e tomar decisões pela equipe ${ }^{44}$. Observou-se, portanto, um nível de controle entre motoristas que se sobrepõe ao dos coletores.

Meyer et al. ${ }^{10}$, verificaram os maiores escores do JCQ para demanda psicológica e os menores para controle sobre o trabalho entre trabalhadores negros comparados com brancos. No entanto, os autores utilizaram menos itens do JCQ que o presente estudo, dificultado a comparação. Existe uma lacuna na produção científica sobre a distribuição étnica das exposições ocupacionais, o que limita a discussão dos resultados obtidos com base em outras evidências.

Contudo, há achados na literatura convergentes com os do presente estudo, inclusive com maiores magnitudes de associação entre raça/cor e demanda psicossocial, obtidos entre profissionais de enfermagem norte-americanos ${ }^{22}$. Os negros apresentaram o dobro de baixo controle e quase o triplo do trabalho de alta exigência, após ajuste por variáveis sociodemográficas e ocupacionais. Ao estratificar por categorias de atuação, a frequência de alta exigência ampliou-se entre auxiliares negros, porém não houve disparidades por raça/cor entre os profissionais mais qualificados.
Estudo que verificou maior prevalência de alta demanda psicológica entre amarelos (54,8\%), seguidos por brancos $(51,2 \%)$, pardos $(49,9 \%)$ pretos $(48,8 \%)$ e indígenas $(41,8 \%)$, ao mesmo tempo mostrou que a proporção de elevado grau de controle no trabalho foi observado na mesma ordem $^{23}$. Portanto, as frequências de alta demanda e alto controle entre amarelos e brancos observadas pelos autores condizem com o trabalho ativo, a experiência mais favorável segundo o Modelo D-C, que se relaciona com motivação, elevação da autoestima e desenvolvimento de novas habilidades para o trabalho ${ }^{20}$. Alta demanda psicológica e baixo controle no trabalho, por sua vez, mais frequentes entre pretos, configuram o trabalho de alta exigência, consistente com nossos achados. De modo semelhante, outros autores $^{24}$ encontraram que a alta exigência foi superior entre não-brancos $(28,1 \%)$ quando comparados com brancos $(21,6 \%)$.

As médias de demanda psicológica e autoridade para decisão encontradas por Thomas et al. ${ }^{45}$ através do JCQ, no entanto, foram semelhantes entre os grupos raciais estudados. Outros autores $^{46}$ também não encontraram diferenças nas demandas psicossociais no trabalho conforme raça/cor, em estudo com trabalhadores com idade igual ou superior a 50 anos, no entanto, esses autores não utilizaram o MDC-AS, nem o JCQ, restringindo a comparação dos resultados.

O apoio social advindo de supervisores foi mais baixo entre os pretos. Sabe-se que o apoio social proveniente de supervisores tende a ser mais protetor frente a situações de trabalho com alta demanda psicológica, haja vista o papel de supervisores na gestão e organização do trabalho ${ }^{47}$. O baixo apoio social de supervisor pode contribuir desta forma para condições de sofrimento psíquico e físico, incluindo a dor musculoesquelética ${ }^{48}$.

Por outro lado, o maior apoio social proveniente de colegas verificado entre pretos pode indicar maior solidariedade entre esses trabalhadores, provavelmente como estratégia de enfrentamento à maior exposição a condições adversas no trabalho. Por isso, o apoio social é também referido como controle coletivo ${ }^{22,47}$. No entanto, o baixo apoio social, combinando apoio de colegas e supervisor, predominou entre os pretos e pardos, comparados aos brancos. Em situações de elevada demanda psicológica, de baixo controle do trabalhador para regular o ritmo e a carga de trabalho e de baixo apoio social, a adoção de pausas durante a jornada, a flexibilização das posturas corporais, a fim de evitar sobrecarga física e mental, estão comprometidas ${ }^{47}$. 
Iniquidades raciais foram também constatadas quanto à demanda física no trabalho, revelando uma sobreposição de desvantagens entre indivíduos negros. O trabalho com os braços elevados foi mais frequente entre estes. Esta postura reduz o espaço subacromial no ombro, podendo ocasionar dor incapacitante ${ }^{27,30,49,50}$. Frequentemente a elevação de braços ocorre com manuseio de carga simultaneamente ${ }^{27,49}$, como quando coletores lançam cargas em caminhão de coleta do lixo ${ }^{44,51}$.

Manuseio de carga e agachamentos, que têm sido associados à sintomatologia musculoesquelética ${ }^{30,31,50}$, ocorreram em maior proporção entre negros - pardos e pretos - podendo resultar de tarefas desenvolvidas por coletores de lixo urbano.

Enquanto levantar materiais associa-se principalmente com lombalgia, tarefas que envolvem empurrar e puxar frequentemente relacionamse com dor em região cervical e em ombros ${ }^{30,50}$. Schibye et al. ${ }^{26}$, ao simularem em laboratório tarefas realizadas por coletores de lixo urbano, verificaram aumento das forças compressivas e de cisalhamento sobre a coluna vertebral ao levantar materiais e ao empurrar e puxar contêiner sobre duas rodas.

Alta exposição ao manuseio de materiais ou a posturas anômalas no trabalho foi associada ao absenteísmo de longa duração $0^{52} \mathrm{e}$ ao aumento de $50 \%$ de dor em múltiplos locais do corpo nos últimos sete dias $^{33}$ e esta maior morbidade se relaciona com maiores prevalências de limitação para o trabalho ${ }^{33}$.

Observou-se uma menor proporção de pretos nos cargos de coordenação, supervisão ou administrativos, que em geral favorecem a adoção de diferentes posturas de trabalho e pouco manuseio de carga. Por outro lado, alta exposição dos pretos ao trabalho em pé mantido, que é muito penoso, pode resultar em lombalgia e edema em membros inferiores ${ }^{29}$. A exigência de postura em pé para cumprimento das tarefas é descrita nos setores de corte, montagem e acabamento na fabricação de calçados, às vezes, associada à rotação de tronco ${ }^{53}$.

A maior frequência do manuseio de carga e de posturas anômalas entre pretos e pardos suscita a discussão sobre o período escravagista, quando os corpos dos negros eram utilizados para o transporte de cargas. Desta forma, o trabalho físico pesado pode representar a perpetuação do racismo que se explicita pela desigualdade de exposição no trabalho ${ }^{6,14}$.

Os gradientes de exposição verificados entre pretos e pardos sugerem, em acordo com a litera- tura $^{54}$, que indivíduos com características negras mais acentuadas, como o tom de pele mais escuro, que provavelmente se autoclassificam pretos, estão mais vulneráveis que aqueles com pele menos escura. Estes, contudo, persistem em desvantagem em comparação com os brancos. Portanto, os gradientes expressos no presente estudo pelas razões de prevalência poderiam ser maiores se não fosse a miscigenação característica, principalmente, da cidade de Salvador. Ao mesmo tempo, a narrativa da miscigenação como mecanismo de democratização racial tem contribuído para ocultação das iniquidades ${ }^{6}$.

A menor escolaridade entre negros, tal como observado na caracterização da população de estudo, é possivelmente uma das manifestações do racismo estrutural, que inclui a naturalização de iniquidades intergeracionais de posição econômica e refletem o passado de escravização, embora não seja consequência exclusiva e irreversível desse histórico ${ }^{6,14}$. Apesar desta característica encontrada na população estudada, a associação entre raça/cor e demanda no trabalho, física ou psicossocial, foi ajustada pela escolaridade, conforme pode ser constatado nos modelos de análise, além do ajuste por sexo e idade. Os achados evidenciam que trabalhadores pretos, portanto, estão destinados aos postos de trabalho que implicam maior sobrecarga física e psíquica, comparados com os trabalhadores brancos, de mesma escolaridade, sexo e idade, ratificando o papel da raça/cor na exposição ocupacional.

O presente estudo apresenta como contribuições a abordagem de duas modalidades centrais de exposição ocupacional - exposição às demandas físicas e às demandas psicossociais - pouco exploradas na literatura científica quanto às iniquidades raciais em sua distribuição.

Estudar 1.032 trabalhadores dos setores da indústria e de serviços, em coleta de dados presencial, no mundo privado do trabalho, é uma superação de barreira de acesso de pesquisadores a esta população. Estudos epidemiológicos em Saúde do Trabalhador têm se restringido, muitas vezes, a populações de servidores públicos ou população geral, na qual se tenta especificar "ocupação" e as contribuições do trabalho, com todas as limitações de validade conhecidas nessas circunstâncias. Ademais, a variabilidade na população do estudo quanto às ocupações favoreceu, ao mesmo tempo, a variabilidade na variável raça/cor.

Os procedimentos adotados podem representar pontos-fortes do estudo. A garantia de privacidade e confidencialidade para obtenção das 
informações nos locais de trabalhos, considerando a possibilidade de conflitos de interesse entre trabalhadores e empregadores; a independência dos pesquisadores frente às empresas; a coleta conduzida por meio de entrevista por pesquisadores vinculados à instituição pública de ensino, possibilitaram minimizar o viés de informação e ampliar a taxa de resposta no estudo. A estratégia de seleção dos participantes contribuiu para reduzir a possibilidade de viés de sobrevivência do trabalhador sadio.

A variável raça/cor pode apresentar erros de classificação, transitoriedade ao longo do tempo e imprecisão conceitual para estudos em saú$\mathrm{de}^{17-20}$. No entanto, procedeu-se à análise epidemiológica conforme o uso recomendado desta variável na literatura. A decisão metodológica de exclusão de amarelos e indígenas foi justificada com base no número de indivíduos dessas etnias na população estudada, no entanto, vale destacar a lacuna na literatura sobre exposições ocupacionais nesses grupos étnicos e a inserção precária de indígenas, principalmente, no mercado de trabalho?.

\section{Colaboradores}

JS Siqueira e RCP Fernandes participaram equitativamente da concepção do estudo, da análise e interpretação dos dados, da redação do artigo, da revisão crítica do conteúdo intelectual e da aprovação da versão final do artigo. São, portanto, responsáveis por todos os aspectos do trabalho na garantia da exatidão e integridade de qualquer parte do artigo.

\section{Conclusão}

A raça/cor de trabalhadores é um marcador de iniquidades no mundo do trabalho, evidenciadas, no presente estudo, através da maior exposição à demanda psicossocial e à demanda física entre pretos e pardos, havendo um gradiente de exposição entre esses quando comparados com brancos.

Destacaram-se, entre os trabalhadores pretos, a maior exposição à alta exigência psicossocial, bem como ao trabalho físico pesado com exigência de postura anômala e com manuseio de cargas. Os pardos também apresentaram baixo controle sobre o trabalho e exposição física a postura anômala, principalmente.

As evidências apresentadas, somadas à literatura disponível, podem contribuir para o debate social acerca das repercussões do racismo estrutural no mundo do trabalho, bem como para formulação de estratégias antirracistas pelas empresas com foco na reestruturação dos ambientes e processos de trabalho, visando à equidade racial. Intervenções a fim de minimizar a exposição de todos os trabalhadores à demanda psicossocial e física e aquelas direcionadas aos processos seletivos e aos mecanismos de mobilidade na estrutura organizacional são algumas possibilidades. 


\section{Referências}

1. Whitehead M. The concepts and principles of equity and health. Health Promot Int 1991; 6(3):217-229.

2. Silva JB, Barros MBA. Epidemiologia e desigualdade: notas sobre a teoria e a história. Rev Panam Salud Publica 2002; 12(6):375-383.

3. Barreto ML. Desigualdades em Saúde: uma perspectiva global. Cien Saude Colet 2017; 22(07):2097-2108.

4. Bailey Z, Krieger N, Agénor M, Graves J, Linos N, Bassett MT. Structural racism and health inequities in the USA: evidence and interventions. Lancet 2017; 389:1453-1463.

5. Williams DR, Lawrence JA, Davis BA. Racism and Health: Evidence and Needed Research. Annu Rev Public Health 2019; 40:14.1-14.21.

6. Almeida S. O que é racismo estrutural. Letramento: Belo Horizonte; 2018.

7. Osório R. O Sistema classificatório de "cor ou raça" do IBGE. Brasília: IPEA; 2003.

8. Brasil. Instituto de Pesquisa e Economia Aplicada (IPEA). Retrato das desigualdades de gênero e raça [Internet]. 2011 [acessado 2019 fev 9]. Disponível em: http://www.ipea.gov.br/retrato/.

9. Instituto Brasileiro de Geografia e Estatística (IBGE). Desigualdades sociais por cor ou raça no Brasil. Rio de Janeiro: IBGE; 2019.

10. Meyer JD. Race-based job discrimination, disparities in job control, and their joint effects on health. Am J Ind Med 2014; 57:587-595.

11. Steege AL, Baron SL, Marsh SM, Menéndez CC, Myers JR. Examining Occupational Health and Safety Disparities Using National Data: A Cause for Continuing Concern. Am J Ind Med. 2014; 57(5):527-538.

12. Chung-Bridges K, Muntaner C, Fleming LE, Lee DJ, Arheart KL, LeBlanc WG, Christ SL, McCollister KE, Caban AJ, Davila EP. Occupational Segregation as a Determinant of US Worker Health. Am J Ind Med 2008; 51(8):555-567.

13. Conselho Nacional de Justiça (CNJ). Censo do Poder Judiciário: Vetores iniciais e dados estatísticos. Brasília: CNJ; 2014.

14. Batista WM, Mastrodi J. Dos fundamentos extraeconômicos do racismo no Brasil. Rev Direito Praxis 2018; 9(4):2332-2359.

15. Cooper R. A note on the biologic concept of race and its application in epidemiologic research. Am Heart J 1984; 108(3 Parte 2):715-723.

16. Chor D, Lima CRA. Aspectos epidemiológicos das desigualdades raciais em saúde no Brasil. Cad Saude Publica 2005; 21(5):1586-1594.

17. Araújo EM, Nascimento C, Hogan VK. A utilização da variável raça/cor em Saúde Pública: possibilidades e limites. Interface (Botucatu) 2009; 13(31):383-394.

18. Kabad JF, Bastos JL, Santos RV. Raça, cor e etnia em estudos epidemiológicos sobre populações brasileiras: revisão sistemática na base PubMed. Physis 2012; 22(3):895-918.

19. Hicken MT. Measurement and modeling of race and health in Brazil: continuing the discussion. Cad Saude Publica 2017; 33(Supl. 1):1-5.

20. Karasek R. Job demands, job decision latitude, and mental strain. Implications for job redesign. Adm Sci Q 1979; 24:285-308.
21. Johnson JV, Hall EM, Theorell T. Combined effects of job strain and social isolation on cardiovascular disease morbidity and mortality in a random sample of the Swedish male working population. Scand J Work Environ Health 1989; 15(4):271-279.

22. Hurtado DA, Sabbath EL, Ertel KA, Buxton OM, Berkman LF. Racial disparities in job strain among American and immigrant long-term care workers. Int Nurs Rev 2012; 59(2):237-244.

23. Juvanhol LL, Melo ECP, Chor D, Fonseca MJM, Rotenberg L, Bastos LS, Mill JG, Griep RH. Association between demand-control model components and blood pressure in the ELSA-Brasil study: exploring heterogeneity using quantile regression analyses. Scand J Work Environ Health 2018; 44(6):601-612.

24. Lopes SV, Silva MC. Estresse ocupacional e fatores associados em servidores públicos de uma universidade federal do sul do Brasil. Cien Saude Colet 2018; 23(11):3869-3880.

25. Stock SR, Fernandes R, Delisle A, Vézina N. Reproducibility and validity of workers' self-reports of physical work demands. Scand J Work Environ Health 2005; 31(6):409-437.

26. Schibye B, Sùgaard K, Martinsen D, Klausen K. Mechanical load on the low back and shoulders during pushing and pulling of two-wheeled waste containers compared with lifting and carrying of bags and bins. Clinical Biomechanics 2001; 16(7):549-559.

27. Pope DP, Silman AJ, Cherry NM, Pritchard C, Macfarlane GJ. Association of occupational physical demands and psychosocial working environment with disabling shoulder pain. Ann Rheum Dis 2001; 60(9):852-858.

28. Pataro SMS, Fernandes RCP. Trabalho físico pesado e dor lombar: a realidade na limpeza urbana. Rev Bras Epidemiol 2014; 17(1):17-30.

29. Antle DM, Cormier L, Findlay M, Miller LL, Côté JN. Lower limb blood flow and mean arterial pressure during standing and seated work: Implications for workplace posture recommendations. Prev Med Reports 2018; 10:117-122.

30. Jakobsen M, Sundstrup E, Brandt M, Persson R, Andersen L. Estimation of physical workload of the low-back based on exposure variation analysis during a full working day among male blue-collar workers. Cross-sectional work place study. Apllied Ergon 2018; 70:127-133.

31. Allen K, Chen J, Callahan L, Golightly Y, Helmick C, Renner J, Schwartz TA, Jordan JM. Racial Differences in Knee Osteoarthritis Pain: Potential Contribution of Occupational and Household Tasks. J Rheumatol 2014; 39(2):337-344.

32. Fernandes RCP, Pataro SMS, Carvalho RB, Burdorf A. The concurrence of musculoskeletal pain and associated work-related factors: A cross sectional study. BMC Public Health 2016; 16(1):1-9.

33. Fernandes RCP, Burdorf A. Associations of multisite pain with healthcare utilization, sickness absence and restrictions at work. Int Arch Occup Environ Health 2016; 89:1039-1046.

34. Karasek R, Gordon G, Piotrowski C. The Job Content Instrument: Questionnaire and User's Guide. Los Angeles: University of Southern California; 1986. 
35. Araújo TM, Karasek R. Validity and reliability of the job content questionnaire in formal and informal jobs in Brazil. Scand J Work Environ Health 2008; 34(6):5259.

36. Santos KOB, Araújo TM, Carvalho FM, Karasek R. The job content questionnaire in various occupational contexts: Applying a latent class model. BMJ Open 2017; 7(5):1-8

37. Fernandes RCP, Cunha LP, Lima, VC; Santos KOB. Mensurando a demanda física no trabalho: estrutura fatorial e confiabilidade de itens sobre posturas, manuseio de carga e repetitividade. Cad Saude Publica 2019; 35(1):1-14.

38. Barros AJD, Hirakata VN. Alternatives for logistic regression in cross-sectional studies: an empirical comparison of models that directly estimate the prevalence ratio. BMC Med Res Methodol 2003; 3(21):1-13.

39. Coutinho LMS, Scazufca M, Menezes PR. Métodos para estimar razão de prevalência em estudos de corte transversal. Rev Saude Publica 2008; 42(6):992-998.

40. LaMontagne AD, Krnjacki L, Kavanagh AM, Bentley R. Psychosocial working conditions in a representative sample of working Australians 2001-2008: An analysis of changes in inequalities over time. Occup Environ Med 2013; 70(9):639-647.

41. Greenland S. Randomization, Statistics, and Causal Inference. Epidemiology 1990; 6(1):421-429.

42. Hahn GJ, Meeker WQ. Assumptions for Statistical Inference. Am Statistician 1993; 47(1):1-11.

43. Daniel WW, Cross CL. Biostatistics: a foundation for analysis in the health sciences. USA: John Wiley; 2013.

44. Camada IMDO, Pataro SMS, Fernandes RCP. Heavy physical work under time pressure: The garbage collection service-a case study. Work 2012; 41(Supl. 1):462-469.

45. Thomas KMS, Nelesen RA, Ziegler MG, Bardwell WA, Dimsdale JE. Job strain, ethnicity, and sympathetic nervous system activity. Hypertension 2004; 44(6):891-896.

46. Mezuk B, Ratliff S. Job Strain, workplace discrimination, and hypertension among older workers: the health and retirement study. Race Soc Probl 2011; $3(1): 38-50$.

47. Luchman JN, González-Morales MG. Demands, control, and support: A meta-analytic review of work characteristics interrelationships. J Occup Health Psychol 2013; 18(1):37-52.
48. Carvalho RLRB, Fernandes RCP, Lima VMC. Demandas psicológicas, baixo apoio social e repetitividade: fatores ocupacionais associados à dor musculoesquelética de trabalhadores da indústria de calçados. Rev Bras Saude Ocup 2019; 44(6):1-11.

49. Kratzenstein S, Wanstrath M, Behrenbruch K. Height adjustments on backpack-carrying systems and muscle activity. Appl Ergon 2019; 74:172-176.

50. -Wollensen A, Wollensen B, Leitner M, Mattes K. Human Body Mechanics of Pusshing pulling: Analyzing the factors of task-related Strain on the musculoeskeletal System. Saf Health Work 2017: 8(1):11-18.

51. Ziaei M, Choobineh A, Abdoli-Eramaki M, Ghaem $\mathrm{H}$, Jaberi O. Psychological and physical job demands, decision latitude, and work-related social support among Iranian waste collectors. Waste Management 2019; 95:377-387.

52. Andersen LL, Fallentin N, Thorsen SV, Holtermann A. Physical workload and risk of long-term sickness absence in the general working population and among blue-collar workers: Prospective cohort study with register follow-up. Occup Environ Med 2016; 73(4):246253.

53. Lourinho MG, Negreiros GR, Almeida, LB, Vieira ER, Quemelo PRV. Riscos de lesão musculoesquelética em diferentes setores de uma empresa calçadista. Fisioter Pesqu 2011; 18(3):252-257.

54. Dixon AR, Telles EE. Skin Color and Colorism: Global Research, Concepts, and Measurement. Annu Rev Sociol 2017; 43:405-424.

Artigo apresentado em 05/02/2020

Aprovado em 13/08/2020

Versão final apresentada em 15/08/2020

Editores-chefes: Romeu Gomes, Antônio Augusto Moura da Silva 